\title{
Prevalence of and Factors Associated with Albuminuria in the Korean Adult Population: The 2011 Korea National Health and Nutrition Examination Survey
}

\author{
Jong Chul Won ${ }^{1}$, Yun Jeong Lee ${ }^{2}$, Jung Min Kim ${ }^{1}$, Sang Youb Han ${ }^{2}$, Jung Hyun Noh${ }^{2}$, Kyung Soo Ko ${ }^{1}$, \\ Byoung Doo Rhee', Dong-Jun Kim ${ }^{2 *}$
}

1 Department of Internal Medicine, Sanggye Paik Hospital, Cardiovascular and Metabolic Disease Center, College of Medicine, Inje University, Seoul, Republic of Korea, 2 Department of Internal Medicine, Ilsan-Paik Hospital, College of Medicine, Inje University, Koyang, Gyeonggi-do, Republic of Korea

\begin{abstract}
Background: Microalbuminuria is associated with increased risk of renal disease and cardiovascular diseases even in nondiabetic subjects. High incidence rates of microalbuminuria have been found in a number of population-based studies. However, the prevalence and risk factors associated with microalbuminuria in the general population in Korea are unclear.

Objectives: The present study was performed to estimate the prevalence of microalbuminuria and investigate the associated risk factors in the general adult population using the Fifth Korea National Health and Nutrition Examination Survey (KNHANES V-2) data from 2011.

Methods: A total of 5,202 participants (mean age, 45.6 years; men, 2,337; women, 2,865) were included in the analysis. Microalbuminuria was evaluated in participants of KNHANES V-2 based on the urine albumin-creatinine ratio. Estimated glomerular filtration rate was calculated using the Modification of Diet in Renal Disease study equation.

Results: The weighted prevalence of microalbuminuria was $5.2 \%(95 \% \mathrm{Cl}, 4.4-6.1)$ in the general population. The prevalence of albuminuria is increased with age. After adjustment for age and sex, the presence of albuminuria was associated with increased waist circumference, systolic and diastolic blood pressure, aspartate aminotransferase, triglyceride, fasting plasma glucose, and the presence of hypertension and diabetes. In logistic regression analyses, older age, female sex, diabetes, hypertension, and serum aspartate aminotransferase were independently associated with the presence of albuminuria.

Conclusion: The prevalence of microalbuminuria was found to be $5.2 \%$, and conventional risk factors for cardiovascular diseases are closely related to the presence of microalbuminuria in Korea. Microalbuminuria may be a useful marker to identify individuals with increased risk of cardiovascular disease.
\end{abstract}

Citation: Won JC, Lee YJ, Kim JM, Han SY, Noh JH, et al. (2013) Prevalence of and Factors Associated with Albuminuria in the Korean Adult Population: The 2011 Korea National Health and Nutrition Examination Survey. PLoS ONE 8(12): e83273. doi:10.1371/journal.pone.0083273

Editor: Harald Mischak, University of Glasgow, United Kingdom

Received September 3, 2013; Accepted November 12, 2013; Published December 27, 2013

Copyright: ( 2013 Won et al. This is an open-access article distributed under the terms of the Creative Commons Attribution License, which permits unrestricted use, distribution, and reproduction in any medium, provided the original author and source are credited.

Funding: This work was supported by the Inje Research and Scholarship Foundation in 2013 (DK). The funders had no role in study design, data collection and analysis, decision to publish, or preparation of the manuscript.

Competing Interests: The authors have declared that no competing interests exist.

*E-mail: djkim@paik.ac.kr

\section{Introduction}

In 2009, the overall prevalence of end-stage renal disease (ESRD) in Korea was reported to be 1,113.6 per million subjects, and the prevalence rate increased by about $12 \%$ per year during 2000-2009. The high prevalence of diabetes and the associated renal complications have been described for these years in Korea [1]. Diabetic nephropathy is the most common cause of ESRD (45.4\% in new patients with ESRD) [2].

Microalbuminuria is an important marker of progression to ESRD as well as increased risk of cardiovascular disease (CVD) and mortality across all levels of glomerular filtration rate (GFR) $[3,4]$. Given these well-established links, there is concern regarding the high prevalence of microalbuminuria in a number of populations worldwide because effective interventions are needed to manage risk factors associated with microalbuminuria. These studies showed that the prevalence of microalbuminuria is increased in subjects with one or more GVD risk factors, such as elevated blood pressure or high triglyceride (TG) concentration and low high-density lipoprotein cholesterol (LDL-C) concentration, indicating that microalbuminuria is an early marker of systemic endothelial dysfunction and may be an important clinical sign of adverse health outcomes even in the general adult population [5]. Therefore, it is necessary to estimate its prevalence and risk factors to prepare effective strategies to attenuate the impact of systemic vascular disease on health care costs and quality of life due to substantial renal disease, particularly given the increases in prevalence of obesity, diabetes, and hypertension in Korea $[1,6]$. However, little information is available to estimate the prevalence of albuminuria and associated risk factors in the Korean population. This study was performed to investigate these 
issues in the general population using the fifth Korea National Health and Nutrition Examination Survey (KNHANES V-2) data from 2011.

\section{Materials and Methods}

\section{Study population and data collection}

This study used data from KNHANES V-2, a national probability survey conducted by the Korean Center for Disease Control for Health Statistics at 192 survey locations to determine the health and nutritional status of the civilian, non-institutionalized Korean population since 1998. The Korean Center for Disease Control conducted a series of KNHANES, in 1998, 2001, 2005, 2007-2009, and 2010-2012. In KNHANES V-2, an annual total of 3,840 households were selected, and urine albumin was first measured starting in 2011. A standardized interview was conducted in the homes of the participants to collect information on demographic variables, family history, medical history, medications used, and a variety of other health-related variables. Participants were chosen using proportional allocation-systemic sampling with multistage stratification (age, sex, and region). This study was approved by the institutional review board of Ilsan Paik Hospital, Republic of Korea (IB-1308-031). After approval of the study proposal, the KNHANES dataset was made available at the request of the investigator. As the dataset did not include any personal information and participants' consent had already been given for the KNHANES, our study was exempt from participant consent.

\section{Health interview, examination, and laboratory tests}

The Health Interview included well-established questions to determine the demographic and socioeconomic characteristics of the subjects including questions on age, education level, occupation, income, marital status, smoking habit, alcohol consumption, exercise, previous and current diseases, and family disease history. Smoking status was divided into three categories: current smoker, ex-smoker, and nonsmoker. Subjects were questioned about whether they exercised with an intensity that left them with slight difficulty in breathing and sweating. Subjects who exercised regularly at a moderate intensity were asked about the frequency at which they exercised per week and the length of time per exercise session. Regular exercise was defined exercising as five or more times per week. Alcohol consumption was assessed by questioning the subjects about their drinking behavior during the month before the interview. Heavy alcohol drinking was categorized as drinking four or more times per week.

Height and weight were obtained using standardized techniques and equipment. Height was measured to the nearest $0.1 \mathrm{~cm}$ using a portable standiometer (Seriter, Bismarck, ND). Weight was measured to the nearest $0.1 \mathrm{~kg}$ using a Giant-150N calibrated balance-beam scale (Hana, Seoul, Korea). Body mass index (BMI) was calculated by dividing weight by the square of height $\left(\mathrm{kg} / \mathrm{m}^{2}\right)$. Systolic and diastolic blood pressure (BP) was measured by standard methods using a sphygmomanometer with the patient in the sitting position. Three measurements were made for all subjects at 5-min intervals; the average of the second and third measurements was used in the analysis. Blood samples were collected in the morning after fasting for at least $8 \mathrm{~h}$ and singlespot urine specimen collected in the first morning void. Analysis of fasting plasma glucose and serum total cholesterol, TG, LDL-C and high-density lipoprotein cholesterol levels were performed by Hitachi Automatic Analyzer 7600 (Hitachi, Tokyo, Japan). Serum gamma-glutamyl transpeptidase (GGT) was measured using an enzymatic colorimetric method and aspartate aminotransferase
Table 1. Weighted demographic and clinical characteristics of the Korean population $\geq 19$ years old in the 2011 KNHANES.

\begin{tabular}{|c|c|}
\hline$N$, (unweighted/weighted) & $5,202 / 32,333,446$ \\
\hline Age (years) & $45.6(44.7-46.5)$ \\
\hline Men (\%) & $52.3(50.9-53.8)$ \\
\hline Current smoking (\%) & $27.3(25.5-29.1)$ \\
\hline Heavy alcohol drinking (\%) & $11.7(6.8-8.7)$ \\
\hline Regular exercise (\%) & $13.2(11.8-14.7)$ \\
\hline WC $(\mathrm{cm})$ & $81.7(81.2-82.1)$ \\
\hline BMI $\left(\mathrm{kg} / \mathrm{m}^{2}\right)$ & $23.8(23.6-23.9)$ \\
\hline Obesity (\%) & $32.9(30.9-34.8)$ \\
\hline Diabetes (\%) & $9.0(8.0-10.1)$ \\
\hline Hypertension (\%) & $26.5(24.8-28.2)$ \\
\hline Systolic BP (mmHg) & $117.7(116.0-118.5)$ \\
\hline Diastolic BP (mmHg) & $76.3(75.9-76.8)$ \\
\hline Serum Creatinine $(\mu \mathrm{mol} / \mathrm{l})$ & 79.6 (70.7-79.6) \\
\hline eGFR, $\mathrm{ml} / \mathrm{min} / 1.73 \mathrm{~m}^{2}$ & $89.1(88.3-89.8)$ \\
\hline ACR $(\mathrm{mg} / \mathrm{g} \mathrm{Cr})$ & $2.34(0.84-6.61)$ \\
\hline FPG $(\mathrm{mmol} / \mathrm{l})$ & $5.4(5.3-5.4)$ \\
\hline Serum LDL-C (mmol/l) & $2.9(2.9-3.0)$ \\
\hline Serum TG (mmol/l) & $1.5(1.5-1.6)$ \\
\hline AST (U/I) & $22.5(22.1-22.9)$ \\
\hline ALT (U/I) & $22.2(21.5-22.9)$ \\
\hline GGT (U/I) & $35.4(33.6-37.3)$ \\
\hline Anti-hypertensive drug (\%) & $14.9(13.7-16.3)$ \\
\hline Anti-lipid drug (\%) & $4.4(3.8-5.0)$ \\
\hline
\end{tabular}

Data are expressed as means with $95 \%$ confidence intervals except for ACR expressed as median with inter-quartile range. WC, waist circumference; $\mathrm{BMI}$, body mass index; BP, blood pressure; eGFR, estimated glomerular filtration rate; $A C R$, albumin-creatinine ratio; FPG, fasting plasma glucose; LDL-C, low-density lipoprotein cholesterol; TG, triglyceride; AST, aspartate aminotransferase; ALT, alanine aminotransferase; GGT, $\gamma$-glutamyltransferase.

doi:10.1371/journal.pone.0083273.t001

(AST) and alanine transaminase (ALT) were measured using the standardized kinetic method (Modular P, Roche Diagnostics, Indianapolis, IN). Obesity was defined as BMI $\geq 25 \mathrm{~kg} / \mathrm{m}^{2}$ [7]. Hypertension was defined as systolic $\mathrm{BP} \geq 140 \mathrm{mmHg}$, diastolic $\mathrm{BP} \geq 90 \mathrm{mmHg}$, or use of antihypertensive medications irrespective of BP. Diabetes was defined as fasting plasma glucose $\geq 7.0 \mathrm{mmol} / \mathrm{l}$, current anti-diabetes medication, or a previous diagnosis of diabetes by a doctor.

\section{Assessment and definition of microalbuminuria and estimation of glomerular filtration rate (eGFR)}

Urine albumin and creatinine concentrations were measured in the same laboratory during all surveys. Serum and urinary concentrations of creatinine were measured using a colorimetric method (Hitachi Automatic Analyzer 7600, Hitachi, Tokyo, Japan). The inter-assay coefficient of variation for serum creatinine was $<1.4 \%$. Urinary albumin was measured in random urine samples using a turbidimetric immunoassay (Hitachi Automatic Analyzer 7600; Hitachi). Laboratory control measures in KNHANES V-2 indicated that these assays are highly reliable with consistently low coefficients of variation (3.1\%). The ratio of urinary albumin to urinary creatinine was reported as 
(a)

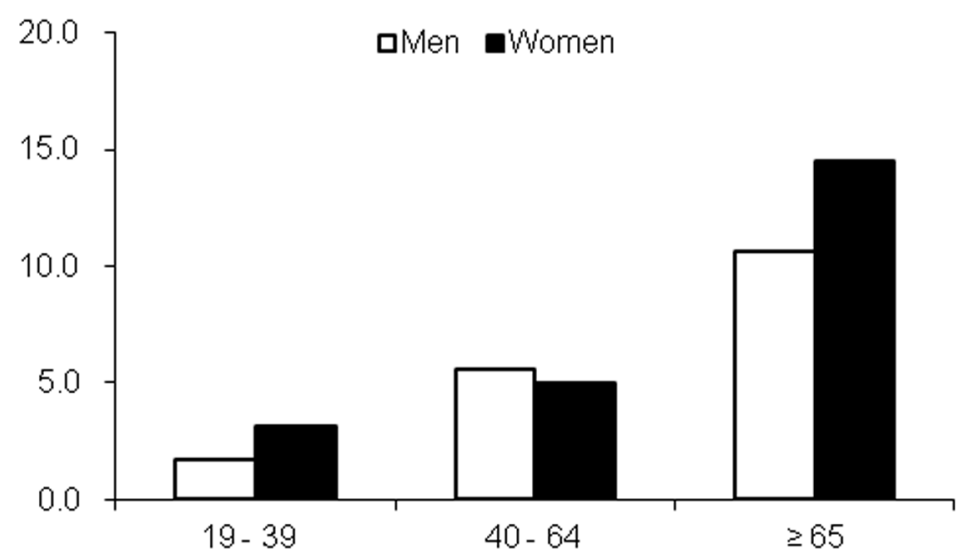

(b)

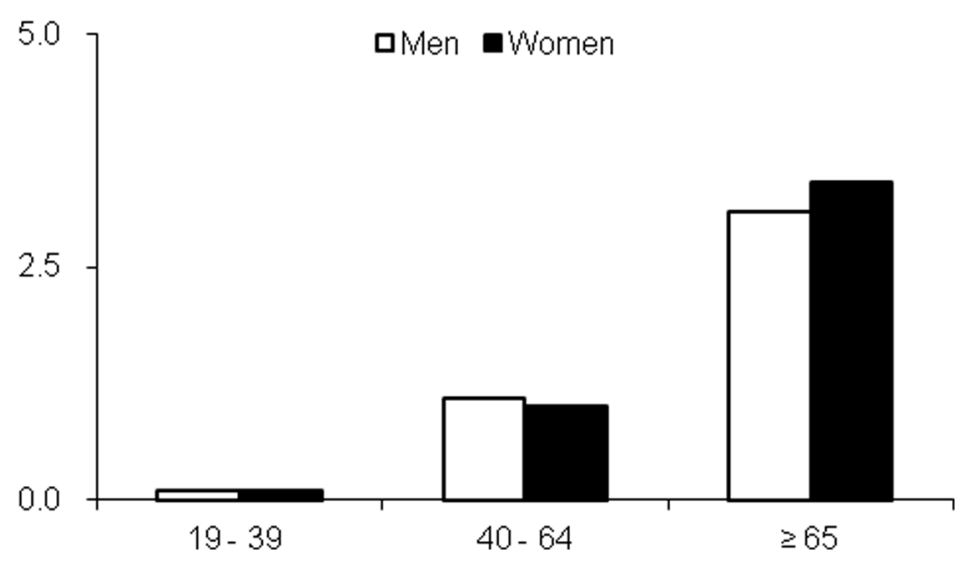

Figure 1. Weighted prevalence (\%) of microalbuminuria (a) and macroalbuminuria (b) by age (years) in the Korean population $\geq 19$ years old in the 2011 KNHANES. Weighted $n$ : men 19-39 years, 7,985,505; 40-64 years, 7,946,568; and $\geq 65$ years, 1,873,929; women, 5,458,591, $7,426,414$, and 2,529,438, respectively.

doi:10.1371/journal.pone.0083273.g001

albumin-creatinine ratio (ACR) in milligrams per gram of creatinine. Subjects were divided according to albuminuria status, i.e., normoalbuminuria (ACR $<20 \mathrm{mg} / \mathrm{g} \mathrm{Cr}$ ), microalbuminuria (ACR $=30-299 \mathrm{mg} / \mathrm{g} \mathrm{Cr})$, or macroalbuminuria (ACR $\geq 300 \mathrm{mg} /$ g Cr) [8]. eGFR was calculated using the abbreviated equation from the Modification of Diet in Renal Disease (MDRD) study: eGFR $\quad\left(\mathrm{ml} / \mathrm{min} / 1.73 \mathrm{~m}^{2}\right)=175 \times\left(\mathrm{S}_{\mathrm{Cr}} / 88.4, \quad \mu \mathrm{mol} /\right.$ 1) ${ }^{-1.154} \times$ Age $^{-0.203} \times 0.742$ (if female) [9]. Additionally, subjects were stratified by eGFR value $\left(\mathrm{ml} / \mathrm{min} / 1.73 \mathrm{~m}^{2}\right)$ into four groups: group 1, eGFR $\geq 90(n=1988)$; group 2, eGFR 60-89 $(n=2981)$; group 3, eGFR 30-59 $(n=215)$; group 4 , eGFR $<30 \mathrm{ml} / \mathrm{min} /$ $1.73 \mathrm{~m}^{2}(n=8)$ [10]. Decreased eGFR was defined as $<60 \mathrm{ml} /$ $\mathrm{min} / 1.73 \mathrm{~m}^{2}$ in this study.

\section{Statistical analyses}

Data are presented as mean and 95\% confidence interval (CI). Participants in Korean NHANES were not sampled randomly. Korean NHANES was designed as a complex, stratified, multistage probability-sampling model. Therefore each participant does not have the same power for representation of whole Korean population. If we try to present prevalence in whole Korean population form the dataset, we should consider how much power each participant for representation (sample weight) for whole Korean population. After approval of investigator's proposal by the Korean Center for Disease Control, the Korean Center for Disease Control provide survey dataset including information about survey location, strata by age, sex, and other factors, and sample weight of each participants to investigator. Survey sample weights, calculated taking the sampling rate, response rate, and age/sex proportion of the reference population (2005 Korean National Census Registry) into consideration, were used in all analyses to produce estimates representative of the non-institutionalized Korean civilian population. Age and age-adjusted comparisons of clinical characteristics according to the presence of microalbuminuria or macroalbuminuria were performed using analysis of covariance (ANCOVA). To determine which parameters were associated with the presence of microalbuminuria or macroalbuminuria, logistic regression analysis was conducted with age, sex, waist circumference, presence of hypertension, serum AST, serum TG, impaired fasting glucose, and diabetes as 
Table 2. Weighted prevalence of albuminuria by age, sex, and eGFR of the Korean population $\geq 19$ years old in the 2011 KNHANES.

\begin{tabular}{|c|c|c|c|c|}
\hline & Normoalbuminuria \% $(95 \% \mathrm{CI})$ & Microalbuminuria, \% (95\% Cl) & Macroalbuminuria \% $(95 \% \mathrm{Cl})$ & \\
\hline Total & $93.8(92.9-94.7)$ & $5.2(4.4-6.1)$ & $1.0(0.7-1.4)$ & $P$ \\
\hline Age, year & & & & $<0.001$ \\
\hline $19-39$ & $97.6(96.3-98.4)$ & $2.3(1.5-3.5)$ & $0.1(0.0-0.4)$ & \\
\hline $40-64$ & $93.6(92.4-94.7)$ & $5.3(4.4-6.5)$ & $1.1(0.6-1.8)$ & \\
\hline$\geq 65$ & $83.9(81.0-86.5)$ & $12.8(10.8-15.2)$ & $3.2(2.1-5.0)$ & \\
\hline Sex & & & & 0.114 \\
\hline Men & $94.5(93.3-95.5)$ & $4.5(3.6-5.7)$ & $0.9(0.6-1.5)$ & \\
\hline Women & $93.1(91.8-94.1)$ & $5.9(4.9-7.1)$ & $1.1(0.7-1.6)$ & \\
\hline eGFR & & & & $<0.001$ \\
\hline$\geq 90$ & $96.1(94.9-96.9)$ & $3.5(2.7-4.6)$ & $0.4(0.2-0.8)$ & \\
\hline $60-89$ & $93.1(91.8-94.2)$ & $5.8(4.8-6.9)$ & $1.1(0.7-1.7)$ & \\
\hline $30-59$ & $73.9(64.7-81.4)$ & $19.0(12.5-27.9)$ & $7.1(4.2-11.8)$ & \\
\hline$<30$ & $33.9(6.5-79.0)$ & $27.8(7.3-65.3)$ & $38.3(12.9-72.2)$ & \\
\hline
\end{tabular}

Data are expressed as estimated prevalence (\%) and 95\% confidence intervals. eGFR, estimated glomerular filtration rate $\left(\mathrm{ml} / \mathrm{min} / 1.73 \mathrm{~m}{ }^{2}\right)$.

doi:10.1371/journal.pone.0083273.t002

variables. Linear regression analysis for $\log$ ACR was performed with age, sex, waist circumference, systolic BP, fasting plasma glucose, serum TG, and serum AST as a continuous variable. Two-tailed analyses were conducted, and $P<0.05$ was deemed to indicate statistical significance. Statistical analyses were performed using SPSS software (ver. 18.0 for Windows; SPSS, Chicago, IL).

\section{Results}

\section{Study population}

A total of 10,589 people participated in the KNHANES V-2, 2011. The participation rate for the health examination (including laboratory tests) was $76.1 \%$. Of these, the 5,202 participants aged over 19 years who completed the measurement of ACR were included in the analysis. Sample data were weighted appropriately to account for the complex design and unequal probability of selection of sample subjects to compute prevalence estimates of abnormal albumin excretion levels for the Korean population.

\section{Demographic and clinical characteristics of the study} population

Table 1 shows the weighted demographic and clinical characteristics of the study population entered into the final analysis. The overall mean age was 45.6 years old (95\% CI, $44.7-$ 46.5). Both sexes were equally represented. The weighted mean age was 44.2 years $(43.2-45.2)$ for men and 47.2 years $(46.2-48.2)$ for women. The weighted mean BMI was $23.8 \mathrm{~kg} / \mathrm{m}^{2}$ (95\% CI, 23.6-23.9), serum creatinine level $\left(\mathrm{S}_{\mathrm{Cr}}\right)$ was $79.6 \mu \mathrm{mol} / \mathrm{l}(95 \% \mathrm{CI}$, 70.7-79.6), and median ACR was $2.34 \mathrm{mg} / \mathrm{g} \mathrm{Cr}$ (inter-quartile, $0.84-6.61$ ). And, $33 \%$ of subjects were obese and $9 \%$ had diabetes. Twenty-seven percent of subjects was hypertension: systolic $\mathrm{BP} \geq 140$ or diastolic $\mathrm{BP} \geq 90 \mathrm{mmHg}$ without antihypertensive medication $(10.8 \%, 563 / 5202)$, or use of antihypertensive medications irrespective of $\mathrm{BP}(21.6 \%, 1124 / 5202)$.

Prevalence of microalbuminuria by age, sex, and eGFR

Table 2 shows the weighted prevalence rates of microalbuminuria and macroalbuminuria according to age, sex, and eGFR. The weighted prevalence rates of microalbuminuria and

Table 3. Prevalence of decreased eGFR (eGFR $<60 \mathrm{ml} / \mathrm{min} / 1.73 \mathrm{~m}^{2}$ ) by age and sex of the Korean population $\geq 19$ years old in 2011 KNHANES.

\begin{tabular}{lll}
\hline & Unweighted prevalence \% & Weighted prevalence \% (95\% Cl) \\
\hline Total & $\mathbf{4 . 3}(\mathbf{2 2 3 / 5 , 2 0 2 )}$ & $\mathbf{2 . 8}(\mathbf{2 . 4 - 3 . 3 )}(\mathbf{9 1 1}, \mathbf{8 0 9 / 3 2 , 3 3 3 , 4 4 6 )}$ \\
\hline Age, year & & \\
$19-39$ & 0.1 & $0.1(0.0-0.8)$ \\
$40-64$ & 2.0 & $2.1(1.5-2.8)$ \\
$\geq 65$ & 13.7 & $13.2(10.9-15.9)$ \\
\hline Gender & & \\
\hline Men & 4.8 & $3.0(2.3-3.7)$ \\
Women & 3.8 & $2.7(2.1-3.4)$ \\
\hline
\end{tabular}

Data are expressed as estimated prevalence (\%) and 95\% confidence intervals.

doi:10.1371/journal.pone.0083273.t003 
Table 4. Weighted number and distribution of eGFR in each category of albuminuria in Korean adult population.

\begin{tabular}{|c|c|c|c|c|c|c|}
\hline \multirow[b]{2}{*}{ eGFR } & \multicolumn{2}{|c|}{ Normoalbuminuria } & \multicolumn{2}{|c|}{ Microalbuminuria } & \multicolumn{2}{|c|}{ Macroalbuminuria } \\
\hline & Weighted $N$ & $\%^{*}$ & Weighted $N$ & $\%^{*}$ & Weighted $N$ & $\%^{*}$ \\
\hline$\geq 90$ & $1,3583,888$ & $44.8(42.4-47.1)$ & 500,437 & $29.9(24.1-36.4)$ & 57,698 & $17.9(9.0-32.5)$ \\
\hline 60-89 & $1,6090,965$ & $53.0(50.8-55.3)$ & 997,347 & $59.6(53.2-65.7)$ & 191,302 & $59.4(44.2-73.0)$ \\
\hline $30-59$ & 653,846 & $2.2(1.7-2.7)$ & 168,312 & $10.1(6.6-15.0)$ & 62,886 & $19.5(11.5-31.3)$ \\
\hline$<30$ & 9,067 & $0.1(0.0-0.2)$ & 7,440 & $0.4(0.1-2.0)$ & 10,257 & $3.2(1.0-9.7)$ \\
\hline
\end{tabular}

*Proportion of each eGFR group. Data are expressed as estimated prevalence (\%) and $95 \%$ confidence intervals. $\chi^{2}$ test, $P<0.001$. eGFR, estimated glomerular filtration rate $\left(\mathrm{ml} / \mathrm{min} / 1.73 \mathrm{~m}^{2}\right)$.

doi:10.1371/journal.pone.0083273.t004

macroalbuminuria were $5.2 \%$ (95\% CI, $4.4-6.1)$ and $1.0 \%(0.7-$ 1.4), respectively. These prevalence rates were significantly increased with age and were high in subjects of both sexes aged $\geq 65$ years $(P<0.001)$. Generally, the prevalence of microalbuminuria or macroalbuminuria was not different according to sex. In subjects $<65$ years old, the prevalence was higher in men. The reverse pattern was observed in subjects $\geq 65$ years old, but the difference was not statistically significant (Figure 1).

The prevalence of decreased eGFR $\left(<60 \mathrm{ml} / \mathrm{min} / 1.73 \mathrm{~m}^{2}\right)$ was $2.8 \%$ (95\% CI, 2.4-3.3) (Table 3). When subjects with microalbuminuria or macroalbuminuria were stratified by eGFR, among subjects with eGFR of $30-59 \mathrm{ml} / \mathrm{min} / 1.73 \mathrm{~m}^{2}$, the prevalence rates of microalbuminuria and macroalbuminuria were $19.0 \%$ (95\% CI, $12.5-27.9)$ and $7.1 \%(4.2-11.8)$, respectively. The rates in cases with eGFR $<30 \mathrm{ml} / \mathrm{min} / 1.73 \mathrm{~m}^{2}$ were $27.8 \%$ (95\% CI, 7.3-65.3) and 38.3\% (12.9-72.2), respectively (Table 2). On the other hand, about $90 \%$ and $80 \%$ of subjects with microalbuminuria and macroalbuminuria, respectively, had relatively preserved eGFR ( $\left.\geq 60 \mathrm{ml} / \mathrm{min} / 1.73 \mathrm{~m}^{2}\right)$ (Table 4$)$.

Table 5. Age-, sex-, and age- and sex-adjusted demographic and clinical characteristics of the Korean population $\geq 19$ years old in the 2011 KNHANES by categories of albuminuria.

\begin{tabular}{|c|c|c|c|c|}
\hline & Normoalbuminuria & Microalbuminuria & Macroalbuminuria & $P$ for trend \\
\hline Number (weighted) & $30,337,766$ & $1,673,536$ & 322,143 & \\
\hline Age (years) & $44.8(44.0-45.7)$ & $57.1(54.6-59.5)$ & $60.4(55.8-64.9)$ & $<0.001$ \\
\hline Women (\%) & $47(46-49)$ & $54(48-61)$ & $50(36-65)$ & 0.126 \\
\hline Current smoking (\%) & $27.1(25.4-28.7)$ & $30.8(24.5-37.0)$ & $27.7(15.5-39.9)$ & 0.510 \\
\hline Heavy alcohol drinking (\%) & $7.8(6.8-8.8)$ & $6.493 .2-9.7)$ & $7.7(1.0-16.7)$ & 0.740 \\
\hline Regular exercise (\%) & $13.4(11.9-14.9)$ & $9.0(5.4-12.6)$ & $14.4(4.0-24.8)$ & 0.079 \\
\hline WC $(\mathrm{cm})$ & $81.5(81.0-82.0)$ & $84.6(82.8-86.3)$ & $84.5(81.8-87.2)$ & $<0.001$ \\
\hline Obesity (\%) & $32.3(30.4-34.3)$ & $41.0(33.2-48.9)$ & $38.5(24.1-53.0)$ & 0.066 \\
\hline Diabetes (\%) & $7.9(6.9-8.8)$ & $23.5(17.5-29.6)$ & $38.6(24.6-52.6)$ & $<0.001$ \\
\hline Hypertension (\%) & $24.8(23.3-26.3)$ & $50.9(43.4-58.4)$ & $54.8(41.6-68.0)$ & $<0.001$ \\
\hline Systolic BP (mmHg) & $117.1(116.5-117.7)$ & $125.9(123.3-128.5)$ & $134.1(128.5-139.7)$ & $<0.001$ \\
\hline Diastolic BP (mmHg) & $76.1(75.7-76.5)$ & $79.5(78.0-81.1)$ & $80.2(76.3-84.0)$ & $<0.001$ \\
\hline Serum Creatinine $(\mu \mathrm{mol} / \mathrm{l})$ & $75.1(74.3-76.0)$ & $76.9(75.1-79.6)$ & $88.4(81.3-96.4)$ & 0.001 \\
\hline eGFR, $\mathrm{ml} / \mathrm{min} / 1.73 \mathrm{~m}^{2}$ & $89.1(88.4-89.9)$ & $88.9(86.4-91.3)$ & $81.2(75.6-86.7)$ & 0.023 \\
\hline ACR $(\mathrm{mg} / \mathrm{g} \mathrm{Cr})$ & $2.14(0.77-4.66)$ & $60.35(40.73-114.93)$ & $575.30(403.95-977.42)$ & $<0.001$ \\
\hline FPG $(\mathrm{mmol} / \mathrm{l})$ & $5.3(5.3-5.4)$ & $5.8(5.6-5.9)$ & $6.9(6.0-7.8)$ & $<0.001$ \\
\hline Serum LDL-C (mmol/l) & $2.9(2.9-3.0)$ & $3.0(2.8-3.2)$ & $2.9(2.0-3.7)$ & 0.867 \\
\hline Serum TG $(\mathrm{mmol} / \mathrm{l})$ & $1.5(1.5-1.6)$ & $1.7(1.6-1.9)$ & $2.6(1.8-3.4)$ & $<0.001$ \\
\hline AST (U/I) & $22.4(22.0-22.8)$ & $24.8(22.7-26.8)$ & $25.2(20.9-29.5)$ & 0.023 \\
\hline ALT (U/l) & $22.1(21.4-22.7)$ & $24.7(21.8-27.6)$ & $22.2(18.2-26.1)$ & 0.185 \\
\hline GGT (U/I) & $35.1(33.2-36.9)$ & $41.7(31.6-51.8)$ & $39.3(26.7-51.8)$ & 0.367 \\
\hline Anti-hypertensive drug (\%) & $13.8(12.7-15.0)$ & $30.7(23.8-37.7)$ & $36.9(22.6-51.1)$ & $<0.001$ \\
\hline Anti-lipid drug (\%) & $4.3(3.7-5.0)$ & $6.0(2.7-9.2)$ & $4.3(1.0-10.7)$ & 0.640 \\
\hline
\end{tabular}

Data are expressed as means with $95 \%$ confidence intervals except for ACR expressed as median with inter-quartile range. WC, waist circumference; BMI, body mass index; BP, blood pressure; eGFR, estimated glomerular filtration rate; ACR, albumin-creatinine ratio; FPG, fasting plasma glucose; LDL-C, low-density lipoprotein cholesterol; TG, triglyceride; AST, aspartate aminotransferase; ALT, alanine aminotransferase; GGT, $\gamma$-glutamyltransferase.

doi:10.1371/journal.pone.0083273.t005 
(a)

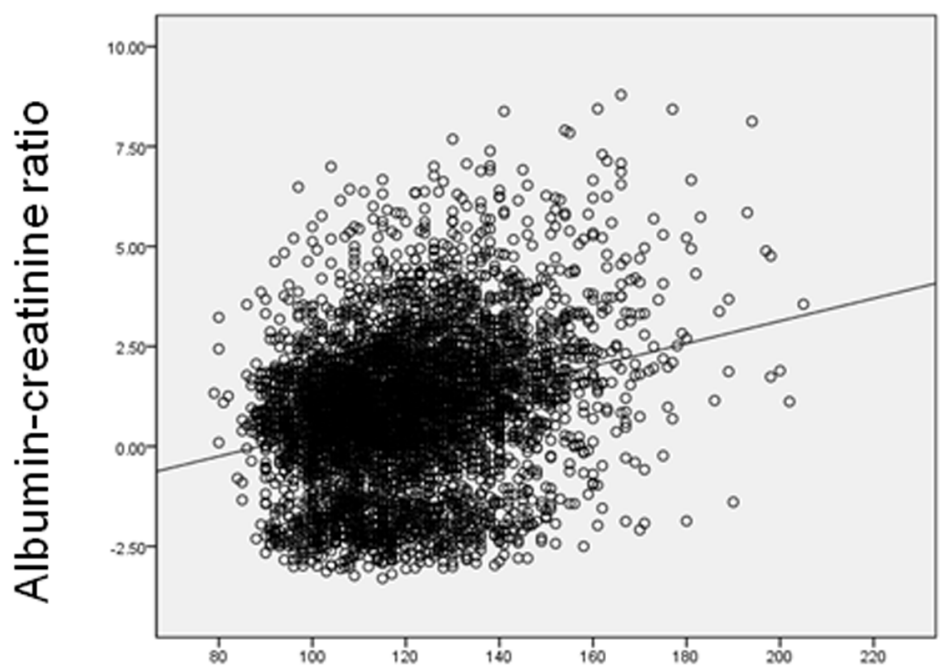

\section{Systolic blood pressure}

(b)

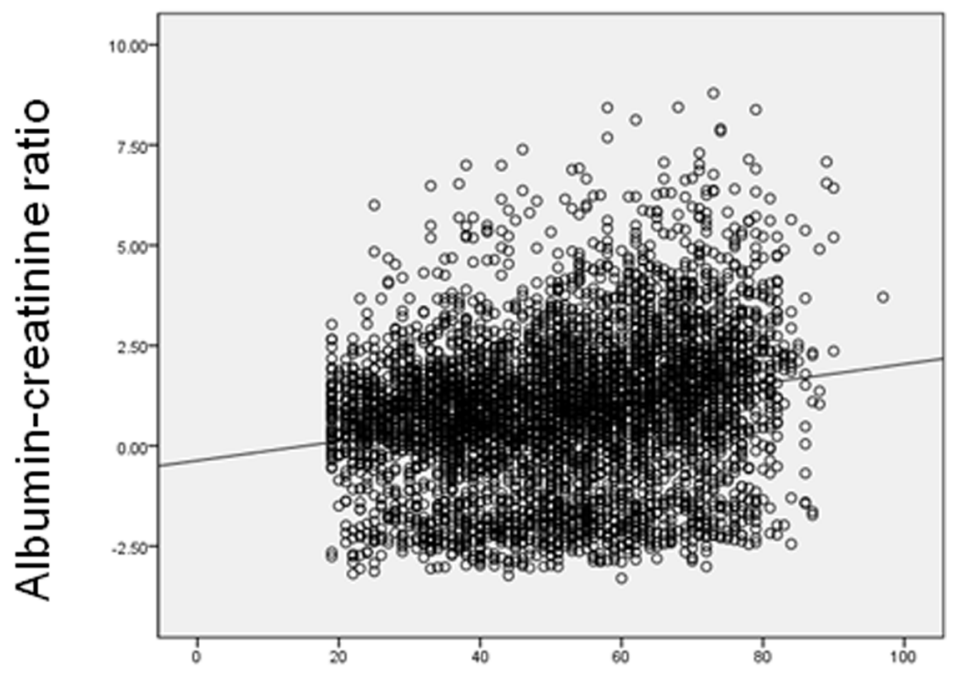

\section{Age}

Figure 2. The relationship between the albumin-creatinine ratio $(\mathrm{mg} / \mathrm{g} \mathrm{Cr}$, log transformed) versus systolic blood pressure (mmHg) (a, $r=0.267, P<0.001)$ and age (yr) (b, $r=0.214, P<0.001)$.

doi:10.1371/journal.pone.0083273.g002

Clinical characteristics of the adult population according to the presence of albuminuria

Table 5 summarizes age-, sex-, and age- and sex-adjusted clinical characteristics of the adult population according to the presence of microalbuminuria and macroalbuminuria. The prevalence of albuminuria increased with age. There were no differences in the presence of albuminuria by sex. After simultaneously controlling for age and sex, results showed that waist circumference, systolic and diastolic BP, AST, TG, fasting plasma glucose, and the presence of hypertension and diabetes were significantly different among three groups. However, there was no difference in current smoking, heavy alcohol drinking, regular exercise, ALT, GGT, LDL-C, or proportion of subjects taking lipid-lowering drugs.

\section{Factors associated with albuminuria}

Multiple logistic regression analysis showed that age, female sex, AST, hypertension, and diabetes were independently associated 
Table 6. Multiple logistic regression analyses for albuminuria or macroalbuminuria.

\begin{tabular}{|c|c|c|c|c|}
\hline & \multicolumn{2}{|l|}{ Albuminuria $^{a}$} & \multicolumn{2}{|c|}{ Macroalbuminuria ${ }^{b}$} \\
\hline & OR $(95 \% \mathrm{Cl})$ & $P$ & OR $(95 \% \mathrm{Cl})$ & $P$ \\
\hline \multicolumn{5}{|l|}{ Age (year), 19-39 } \\
\hline $40-64$ & $1.47(0.95-2.27)$ & 0.087 & $4.68(1.15-19.08)$ & 0.031 \\
\hline$\geq 65$ & $2.57(1.57-4.22)$ & $<0.001$ & $9.42(2.47-35.94)$ & 0.001 \\
\hline Men & $0.65(0.49-0.85)$ & 0.002 & $0.82(0.46-1.48)$ & 0.505 \\
\hline \multicolumn{5}{|l|}{ Waist circumference } \\
\hline per $10 \mathrm{~cm}$ increase & $1.17(0.99-1.38)$ & 0.071 & $1.04(0.76-1.43)$ & 0.791 \\
\hline Hypertension & $3.24(2.25-4.66)$ & $<0.001$ & $2.94(1.37-6.30)$ & 0.006 \\
\hline \multicolumn{5}{|l|}{ Normal fasting glucose } \\
\hline IFG & $1.10(0.75-1.62)$ & 0.613 & $0.57(0.21-1.54)$ & 0.270 \\
\hline Diabetes & $2.85(1.97-4.15)$ & $<0.001$ & $3.54(1.82-6.87)$ & $<0.001$ \\
\hline \multicolumn{5}{|l|}{ Serum TG } \\
\hline per $0.2 \mathrm{mmol} / \mathrm{l}$ increase & $1.02(1.00-1.04)$ & 0.060 & $1.06(1.03-1.10)$ & $<0.001$ \\
\hline \multicolumn{5}{|l|}{ Serum AST } \\
\hline per $5 \mathrm{U} / \mathrm{l}$ increase & $1.05(1.01-1.09)$ & 0.020 & $1.02(0.91-1.14)$ & 0.694 \\
\hline
\end{tabular}

with the presence of any albuminuria including micro- and macroalbuminuria (Table 6). Age, hypertension, TG, and diabetes were independently associated with the presence of macroalbuminuria. Linear regression analysis for log ACR was performed with age, sex, waist circumference, systolic BP, fasting plasma glucose, serum TG, and serum AST as a continuous variable (Figure 2). In this model, age, female sex, systolic BP, fasting plasma glucose, and serum AST was independently associated with log transformed ACR (Table 7).

\section{Discussion}

These survey data from a representative sample of the Korean population indicated a microalbuminuria prevalence rate of $5.2 \%$ (95\% CI, 4.4-6.1). Older age, female sex, hypertension, serum TG, and diabetes are independently associated with the presence of albuminuria in the Korean adult population. Microalbuminuria has been shown to be an early sign of progressive renal failure and increased risk for CVD, not only in diabetic or hypertensive patients but also in non-diabetic and non-hypertensive subjects $[11,12]$. As microalbuminuria reflects increased endothelial permeability and intraglomerular capillary pressure, it may be a useful clinical marker of endothelial dysfunction and vascular dysfunction even in the general population [13,14]. Despite these clinical implications and the increased burden of diabetic and nondiabetic renal disease in Korea [1,2], few population-based screening studies for albuminuria in Korean populations have been performed.

The prevalence of microalbuminuria in this study was slightly lower than those in Australia (6.0\%) [15], Europe (7\%) [11], and that in the National Health and Nutrition Examinations Survey (NHANES) III in the US (7.8\%) [16]. However, our results were consistent with previous reports conducted in general populations without ethnic variation in Korea (5.4\%) and in Japan $(4.6 \%)$ $[17,18]$. These discrepancies may have been due mainly to differences in the populations analyzed, diagnostic criteria, or method used for measurement of microalbuminuria. The prevalence of microalbuminuria increases with age. Additionally, the mean age of our subjects was 45.6 years, which was younger than that in other studies. The proportion of subjects with diabetes or hypertension could also be a potential explanation for these differences. In the present study population, the proportions of subjects with diabetes and hypertension were $9.0 \%$ and $26.5 \%$, respectively. These are similar to the values in a previous nationwide study in the general population in Korea [1]. Additionally, urinary excretion of creatinine can vary with ethnicity, and dietary pattern (i.e., relatively low levels of animal protein in Koreans) affects the urinary excretion of creatinine subsequent to ACR [19-21].

Our results were consistent with previous reports in that some studies have demonstrated an association of microalbuminuria with CVD risk factors, including hypertension, hypertriglyceridemia, impaired fasting glucose, and diabetes [22,23]. It is well known that factors such as hypertension and diabetes are associated with the presence of microalbuminuria [24-26], as was also clearly evident from the results of the present study. Older age is a well-known independent risk factor for microalbuminuria and CKD, even in subjects without diabetes or hypertension $[24,26,27]$. Logistic regression analyses in this study indicated that greater age was associated with microalbuminuria and macroalbuminuria. Additionally, we found that older age was associated with decreased eGFR (Table 3). The prevalence of decreased eGFR was much different between subjects aged 19-39 years and those $\geq 65$ years old $(0.1 \%$ vs. $13.2 \%$, respectively, $P<0.001)$. These findings were in agreement with the observation that aging is associated with senescence and interstitial fibrosis and with renal ischemia due to intrarenal arteriosclerosis and cholesterol emboli

Table 7. Linear regression analyses for albumin-creatinine ratio*

\begin{tabular}{|c|c|c|}
\hline & Coefficient $(95 \% \mathrm{CI})$ & $P$ \\
\hline Age (year) & $0.007(0.004-0.011)$ & $<0.001$ \\
\hline Women & $0.419(0.320-0.519)$ & $<0.001$ \\
\hline WC $(\mathrm{cm})(10 \mathrm{~cm}$ increase) & $0.003(-0.002-0.008)$ & 0.234 \\
\hline Systolic BP (mmHg) ffffpppressurepressHypertension & $0.021(0.018-0.024)$ & $<0.001$ \\
\hline Fasting plasma glucose $(\mathrm{mmol} / \mathrm{l})$ & $0.266(0.226-0.305)$ & $<0.001$ \\
\hline Serum TG (mmol/l) & $0.015(-0.027-0.056)$ & 0.494 \\
\hline Serum AST (U/I) & $0.006(0.001-0.010)$ & 0.009 \\
\hline
\end{tabular}

*Log transformed values of albumin-creatinine ratio were used. WC, waist circumference; BP, blood pressure; TG, triglyceride; AST, aspartate aminotransferase.

doi:10.1371/journal.pone.0083273.t007 
involvement [28]. In this study, we used a single cut-off point ( $\geq 30 \mathrm{mg} / \mathrm{g} \mathrm{Cr}$ ) of albuminuria for men and women because this cut-off point is widely accepted in clinical setting and found that men were less likely to have microalbuminuria compared with women (OR, $0.65 ; 95 \%$ CI, $0.49-0.85, P=0.002$ ). This association was also found in NHANES and AusDiab studies [24,26,27]. However, single cut-off point might overestimate the prevalence in women because urinary creatinine excretion is higher in men compared with women $[29,30]$. Although single cut-off point is widely accepted in clinical setting, further investigations are required to determine the mechanisms underlying the roles of sex hormones in the development of microalbuminuria and the cut-off point for each sex in practice.

Although the association between hypertriglyceridemia and albuminuria, including microalbuminuria, was not statistically significant in multiple logistic regression analysis, it appeared to be a significant independent predictor of macroalbuminuria in this study. With adjustment for age and sex, the significant positive trend was observed between across categories of albuminuria and all components of metabolic syndrome (waist circumference, TG, hypertension, and fasting plasma glucose; all $P<0.001$ ). Insulin resistance was suggested to play a role in the development of microalbuminuria by raising glomerular hydrostatic pressure, increasing renal vascular permeability, aggregating glomerular hyperfiltration, and enhancing renal sodium reabsorption [31]. There are several possible mechanisms linking visceral obesity and microalbuminuria, such as systemic inflammation, adipokines, and activation of the sympathetic nervous system and renin-angiotensin system [32]. Although indices of insulin resistance were not measured in this study, hypertriglyceridemia and elevated AST level, which is indicative of non-alcoholic fatty liver disease as another indication of an insulin-resistant clinical phenotype $[33,34]$, were independently associated with macroalbuminuria and albuminuria, respectively.

Although microalbuminuria has been considered a marker of glomerular injury and an early indicator of progression to renal insufficiency, $64 \%$ of the subjects with decreased eGFR had normal albuminuria in the present study. These findings were consistent with the results of the Framingham Heart Study, in which about only one-quarter of subjects with decreased eGFR $\left(<60 \mathrm{ml} / \mathrm{min} / 1.73 \mathrm{~m}^{2}\right)$ had microalbuminuria (ACR, at least $30 \mathrm{mg} / \mathrm{g} \mathrm{Cr}$ ) [35], and the Takahata Study, in which $20.5 \%$ of Japanese subjects with decreased eGFR had microalbuminuria [36]. Microalbuminuria and decreased GFR were suggested as markers of endothelial dysfunction and renal dysfunction, respec-

\section{References}

1. Lim S, Kim DJ, Jeong I-K, Son HS, Chung CH, et al. (2009) A nationwide survey about the current status of glycemic control and complications in diabetic patients in 2006-The committee of the Korean diabetes association on the epidemiology of diabetes mellitus. Korean Diabetes J 33: 48-57.

2. Jin DC (2011) Current status of dialysis therapy in Korea. Korean J Intern Med 26: $123-131$.

3. Ärnlöv J, Evans JC, Meigs JB, Wang TJ, Fox CS, et al. (2005) Low-Grade Albuminuria and Incidence of Cardiovascular Disease Events in Nonhypertensive and Nondiabetic Individuals The Framingham Heart Study. Circulation 112: 969-975.

4. Hemmelgarn BR, Manns BJ, Lloyd A, James MT, Klarenbach S, et al. (2010) Relation between kidney function, proteinuria, and adverse outcomes. JAMA 303: 423-429.

5. Hillege HL, Fidler V, Diercks GF, van Gilst WH, de Zeeuw D, et al. (2002) Urinary albumin excretion predicts cardiovascular and noncardiovascular mortality in general population. Circulation 106:1777-1782.

6. Belasco AG, Sesso R (2002) Burden and quality of life of caregivers for hemodialysis patients. Am J Kidney Dis 39: 805-812.

7. WHO/IASO/IOTF (2000) The Asia-Pacific Perspective: Redefining Obesity and Its Treatment. Health Communications Australia Melbourne, Australia. tively [37]. However, the mechanisms by which different renal injuries cause glomerular endothelial dysfunction or renal atherosclerosis should be clarified in future studies.

These results of the present study clearly indicated the prevalence of microalbuminuria and its associated factors in Korea. However, this study had some limitations. The first is that a single urine albumin creatinine ratio result was used in this analysis. A single measurement of urine albumin excretion and serum creatinine for calculating ACR and eGFR could result in misleading classifications of albuminuria and CKD stages. However, repeated measurement of these parameters or direct measurement of eGFR is both time consuming and costly and is therefore not feasible for nationwide health examination studies. Additionally, single-point measurement of ACR has been used to predict increased CVD risk [38,39]. Second, in this study, the proportion of subjects taking antihypertensive agents was $14.9 \%$ (95\% CI, 13.7-16.3). These subjects may have been taking different antihypertensive agents, which would influence the appearance of microalbuminuria. However, we did not analyze the effects of various antihypertensive agents on the occurrence of microalbuminuria. Finally, this study did not establish a causal relationship because of its cross-sectional nature. Thus, the possibilities of causal relationships remain to be elucidated by prospective observation of the relationships between risk factors outlined in this study and the development of microalbuminuria.

The results of this population-based study indicated the prevalence of microalbuminuria was slightly lower than those in Western population but comparable to those in Asian population. These may suggest ethnic difference although we do not have the data of direct comparison between each ethnic group. However, the presence of microalbuminuria is a matter of concern because this condition is predictive of progressive deterioration of kidney function and is associated with increased risk of CVD events and death. Therefore, identification and management of relevant risk factors in individual with microalbuminuria is an important step to develop targeted preventive efforts in practice because a growing body of evidence suggests that albuminuria is strongly associated with the risk of adverse outcomes even in general populations.

\section{Author Contributions}

Conceived and designed the experiments: JCW DK. Performed the experiments: JCW DK. Analyzed the data: JCW DK. Contributed reagents/materials/analysis tools: DK. Wrote the paper: JCW DK. Contributed to discussions and reviewed the results: YJL JMK JHN SYH KSK BDR.

8. Keane WF, Eknoyan G (1999) Proteinuria, albuminuria, risk, assessment, detection, elimination (PARADE): a position paper of the National Kidney Foundation. Am J Kidney Dis 33: 1004-1010.

9. Lamb EJ, Tomson CR, Roderick PJ (2005) Estimating kidney function in adults using formulae. Ann Clin Biochem 42: 321-345.

10. AL K, YT A, JC J, TH N (2005) Definition and classification of chronic kidney disease: a position statement from Kidney Disease: Improving Global Outcomes (KDIGO). Kidney Int 67: 2089-2100.

11. de Jong PE, Hillege HL, Pinto-Sietsma SJ, de Zeeuw D (2003) Screening for microalbuminuria in the general population: a tool to detect subjects at risk for progressive renal failure in an early phase? Nephrol Dial Transplant 18: 10-13.

12. Scheven L, Van der Velde M, Lambers Heerspink HJ, De Jong PE, Gansevoort RT (2013) Isolated microalbuminuria indicates a poor medical prognosis. Nephrol Dial Transplant 28:1794-801

13. Asselbergs F, De Boer R, Diercks G, Langeveld B, Tio R, et al. (2004) Vascular endothelial growth factor: the link between cardiovascular risk factors and microalbuminuria? Int J Cardiol 93: 211-215.

14. Imanishi M, Yoshioka K, Konishi Y, Okumura M, Okada N, et al. (1999) Glomerular hypertension as one cause of albuminuria in type II diabetic patients. Diabetologia 42: 999-1005. 
15. Atkins RC, Polkinghorne KR, Briganti EM, Shaw JE, Zimmet PZ, et al. (2004) Prevalence of albuminuria in Australia: The AusDiab kidney study. Kidney Int 66: S22-S24.

16. Jones CA, Francis ME, Eberhardt MS, Chavers B, Coresh J, et al. (2002) Microalbuminuria in the US population: third national health and nutrition examination survey. Am J Kidney Dis 39: 445-459.

17. Choi HS, Sung KC, Lee KB (2006) The prevalence and risk factors of microalbuminuria in normoglycemic, normotensive adults. Clin Nephrol 65: $256-261$.

18. Tanaka S, Takase H, Dohi Y, Kimura G (2013) The prevalence and characteristics of microalbuminuria in the general population: a cross-sectional study. BMC Res Notes 6: 256.

19. Nettleton JA, Steffen LM, Palmas W, Burke GL, Jacobs DR (2008) Associations between microalbuminuria and animal foods, plant foods, and dietary patterns in the Multiethnic Study of Atherosclerosis. Am J Clin Nutr 87: 1825-1836.

20. Kim J (2013) Dairy food consumption is inversely associated with the risk of the metabolic syndrome in Korean adults. J Hum Nutr Diet 26: 171-179

21. Sato M, Hozawa A, Konta T, Shao L, Otani K, et al. (2012) Relationship between dietary intake and microalbuminuria: findings from the Takahata study. Clin Exp Nephrol 16: 147-155.

22. Chen J, Muntner P, Hamm LL, Jones DW, Batuman V, et al. (2004) The metabolic syndrome and chronic kidney disease in US adults. Ann Intern Med 140: $167-174$.

23. Marín R, Rodríguez P, Tranche S, Redón J, Morales-Olivas F, et al. (2006) Prevalence of abnormal urinary albumin excretion rate in hypertensive patients with impaired fasting glucose and its association with cardiovascular diseaseJ Am Soc Nephrol 17: S178-S188.

24. Zhang L, Zhang P, Wang F, Zuo L, Zhou Y, et al. (2008) Prevalence and factors associated with CKD: a population study from Beijing. Am J Kidney Dis 51: 373-384.

25. Farah SE, Wals KT, Friedman IB, Pisacano MA, DiMartino-Nardi J (2006) Prevalence of retinopathy and microalbuminuria in pediatric type 2 diabetes mellitus. J Pediatr Endocrinol Metab 19: 937-942.

26. Coresh J, Astor BC, Greene T, Eknoyan G, Levey AS (2003) Prevalence of chronic kidney disease and decreased kidney function in the adult US population: Third National Health and Nutrition Examination Survey. Am J Kidney Dis 41: 1-12.

27. Imai E, Horio M, Iseki K, Yamagata K, Watanabe T, et al. (2007) Prevalence of chronic kidney disease (CKD) in the Japanese general population predicted by the MDRD equation modified by a Japanese coefficient. Clin Exp Nephrol 11: $156-163$.

28. MacIsaac RJ, Panagiotopoulos S, McNeil KJ, Smith TJ, Tsalamandris G, et al. (2006) Is nonalbuminuric renal insufficiency in type 2 diabetes related to an increase in intrarenal vascular disease? Diabetes Care 29: 1560-1566.

29. Connell SJ, Hollis S, Tieszen KL, McMurray JR, Dornan TL (1994) Gender and the clinical usefulness of the albumin: creatinine ratio. Diabet Med 11:3236.

30. Kesteloot H, Joossens JV (1996) On the determinants of the creatinine clearance: a population study. J Hum Hypertens 10:245-49.

31. Tucker B, Anderson C, Thies R, Collins R, Blantz R (1992) Glomerular hemodynamic alterations during acute hyperinsulinemia in normal and diabetic rats. Kidney Int 42: 1160-1168.

32. Bastard J-P, Maachi M, Lagathu C, Kim MJ, Caron M, et al. (2006) Recent advances in the relationship between obesity, inflammation, and insulin resistance. Eur Cytokine Netw 17: 4-12.

33. Kim Y, Kim C-H, Choi C, Chung Y, Lee M, et al. (2001) Microalbuminuria is associated with the insulin resistance syndrome independent of hypertension and type 2 diabetes in the Korean population. Diabetes Res Clin Pract 52: 145-152.

34. Hanley AJ, Williams K, Festa A, Wagenknecht LE, D’Agostino RB, et al. (2005) Liver Markers and Development of the Metabolic Syndrome The Insulin Resistance Atherosclerosis Study. Diabetes 54: 3140-3147.

35. Foster MC, Hwang S-J, Larson MG, Parikh NI, Meigs JB, et al. (2007) Crossclassification of microalbuminuria and reduced glomerular filtration rate: associations between cardiovascular disease risk factors and clinical outcomes. Arch Intern Med 167: 1386.

36. Konta T, Hao Z, Abiko H, Ishikawa M, Takahashi T, et al. (2006) Prevalence and risk factor analysis of microalbuminuria in Japanese general population: the Takahata study. Kidney Int 70: 751-756.

37. Levey AS, de Jong PE, Coresh J, El Nahas M, Astor BC, et al. (2010) The definition, classification, and prognosis of chronic kidney disease: a KDIGO Controversies Conference report. Kidney Int 80: 17-28.

38. Son J-W, Jang E-H, Kim M-K, Kim H-L, Baek K-H, et al. (2012) Usefulness of Albuminuria as Predictor for Coronary Artery Stenosis, Regardless of Estimated Glomerular Filtration Rate, in Patients With Type 2 Diabetes Mellitus. Am J Cardiol 15:1434-1439

39. Borch-Johnsen K, Feldt-Rasmussen B, Strandgaard S, Schroll M, Jensen JS (1999) Urinary albumin excretion an independent predictor of ischemic heart disease. Arterioscler Thromb Vasc Biol 19: 1992-1997. 